\title{
A common mutation in the COG7 gene with a consistent phenotype including microcephaly, adducted thumbs, growth retardation, VSD and episodes of hyperthermia
}

\author{
Eva Morava ${ }^{1,7}$, Renate Zeevaert ${ }^{2,7}$, Eckhard Korsch $^{3}$, Karin Huijben ${ }^{4}$, Suzan Wopereis ${ }^{4}$, \\ Gert Matthijs $^{2}$, Kathelijn Keymolen ${ }^{5}$, Dirk J Lefeber ${ }^{4}$, Linda De Meirleir ${ }^{6}$ and Ron A Wevers ${ }^{4}$ \\ ${ }^{1}$ Department of Pediatrics, Radboud University Nijmegen Medical Centre, Nijmegen, The Netherlands; ${ }^{2}$ Department of \\ Human Genetics, University of Leuven, Leuven, Belgium; ${ }^{3}$ Pediatric Hospital, Amsterdamerstrasse, Köln, Germany; \\ ${ }^{4}$ Laboratory of Pediatrics and Neurology, Radboud University Nijmegen Medical Centre, Nijmegen, The Netherlands; \\ ${ }^{5}$ Medical Genetics, Free University Brussels, Brussels, Belgium; ${ }^{6}$ Pediatric Neurology and Metabolics, Free University \\ Brussels, Brussels, Belgium
}

We describe the clinical and biochemical characteristics in three patients from two different families diagnosed with Congenital Disorder of Glycosylation type Ile owing to a defect in Conserved Oligomeric Golgi complex (COG)7; one of the eight subunits of the $\mathrm{COG}$. The siblings and an unrelated single child of consanguineous parents presented with growth retardation, progressive, severe microcephaly, hypotonia, adducted thumbs, feeding problems by gastrointestinal pseudo-obstruction, failure to thrive, cardiac anomalies, wrinkled skin and episodes of extreme hyperthermia. A combined disorder in the biosynthesis of $\mathrm{N}$ - and O-linked glycosylation with hyposialylation was detected. Western blot analysis showed a severe reduction in the COG5 and 7 subunits of the COG. A homozygous, intronic splice site mutation $(c .169+4 A>C)$ of the COG7 gene was identified in all patients. The phenotype is similar to that previously described in two patients of North African ethnicity with the same mutation, except for the lack of skeletal anomalies and only a mild liver involvement in our patients. We suggest performing protein glycosylation studies and Western blot for the different COG subunits in patients with progressive microcephaly, growth retardation, hypotonia, adducted thumbs and cardiac defects, especially in association with skin anomalies or episodes of hyperthermia. The presence of the characteristic phenotype might warrant direct DNA analysis.

European Journal of Human Genetics (2007) 15, 638-645. doi:10.1038/sj.ejhg.5201813; published online 14 March 2007

Keywords: Congenital Disorders of Glycosylation; microcephaly; adducted thumbs; hyperthermia; COG; lethality

Correspondence: Dr E Morava, Department of Pediatrics, Radboud University Nijmegen Medical Centre, PO Box 9101, 6500 HB Nijmegen, The Netherlands. Tel: + 3124 3619470; Fax: + 3124 3616428,

E-mail: e.morava@cukz.umcn.nl

${ }^{7}$ These authors contributed equally to this work.

Received 22 September 2006; revised 8 February 2007; accepted 8 February 2007; published online 14 March 2007
Introduction

Congenital Disorders of Glycosylation (CDG) are a group of inherited, multisystem disorders caused by different defects in the biosynthesis of glycoconjugates. The first patients were described by Jaeken et al. ${ }^{1}$ Since then numerous different defects have been reported. The first 
defect in one of the subunits of the Conserved Oligomeric Golgi (COG) complex was identified in 2004. ${ }^{2}$

This multi protein complex consists of eight subunits, which are required for the proper structure and function of the Golgi system. ${ }^{3,4}$ The function of the COG system includes the preservation of the integrity of the Golgi, protein transport within the Golgi and between the endoplasmic reticulum (ER) and Golgi and the docking of glycosylation enzymes. ${ }^{5}$

Defects in the COG complex result in multiple deficiencies in protein glycosylation. ${ }^{5,6}$ The different subunits form stable subcomplexes (COG2 together with 3 and 4 forms lobe A and COG5 together with 6 and 7 forms lobe B), where COG1 plays a role in the mediation of the Golgi complex association and COG8 helps the two subcomplexes to assemble into the complete COG complex. ${ }^{6}$

The COG7 subunit was shown to be clinically deficient in two sibling suffering from a generalized protein glycosylation disorder presenting as a congenital multisystem disease. ${ }^{2,7}$ They showed a phenotype of dysmorphic facial features, microcephaly, adducted thumbs, skeletal anomalies, wrinkled skin, VSD, failure to thrive and a severe liver disease. Both siblings died at the age of a few weeks. Biochemical investigations showed increased lysosomal enzyme activities in plasma and a type 2 plasma transferrin isoelectric focusing (TIEF) pattern. O-glycosylation studies by isoelectric focusing of plasma apolipoproteinC-III (ApoC-III) showed increased hyposialylated isoforms. ${ }^{7}$ The patients were found to have a homozygous point mutation IVS $1+4 \mathrm{~A}>\mathrm{C}$ in a splice site of the COG7 gene. ${ }^{2}$ Here, we report on three new patients with a COG defect and a similar phenotype.

\section{Patients and methods Patients}

Patient 1 was born at term as the second female child of healthy, consanguineous parents (first cousins) of Maroccan origin. The pregnancy was complicated with oligohydramnion and placenta insufficiency. At birth weight was $2645 \mathrm{~g}$ (15th centile), length $44 \mathrm{~cm}(<3$ rd centile), and head circumference $31.5 \mathrm{~cm}$ ( $<3 \mathrm{rd}$ centile). Dysmorphic features were noted including upslanting and short palpebral fissures, a narrow and flat forehead with hypertrichosis, a flat malar region, a wide, and short nose, a smooth philtrum, a small mouth, retrognathia, thick gums, low-set, posterior-rotated ears and a short neck with wrinkled skin (Figure 1). Intermittent strabismus convergens was noted. She had adducted thumbs, overlapping, long fingers, a simian crease, contractures of the PIP and DIP joints with ulnar deviation of the hands. The extension movements in the hips and the knees and the flexion/extension of the elbows were slightly limited. She had a decreased muscle mass and muscle tone with decreased deep tendon reflexes. The newborn needed further hospitalization owing to recurrent episodes of hypoglycemia, pulmonary hypertension and muscle hypotonia. She received tube feeding for gastrointestinal pseudoobstruction. Periods of extreme hyperthermia $\left(>41^{\circ} \mathrm{C}\right)$ of unknown origin were observed. Repeated cranial ultrasound examination showed brain atrophy with cerebellar atrophy, corpus callosum hypoplasia, wide cerebral ventricles and mega-cisterna magna. Brain MRI did not show evidence for abnormal gyration. Ultrasound examination of muscles showed lower echodensity comparable with the picture seen in myopathy. The skeletal survey, liver ultrasound and ophthalmologic examination were normal. The echocardiogram detected a perimembranous VSD with ASD II and tricuspidal insufficiency. BERA evaluation confirmed a sensori-neural hearing loss. On EEG, there were paroxysmal changes with multi-focal sharp waves and generalized frontal activity (Table 1).

Serology for perinatal infections was negative. By further screening, laboratory investigations neither signs of kidney or liver dysfunction nor endocrine or metabolic anomalies were found. During the course of the disease, the ASAT and ALAT levels became gradually elevated up to $890 \mathrm{U} / 1$ without signs of cholestasis or icterus. The serum amino acids, acyl-carnitines profile, very long chain fatty acids, 7-dehydrocholesterol and urine organic acids were also normal, except for an elevated serum alanine level $(820 \mathrm{mmol} / \mathrm{l}, \mathrm{C}:<450 \mathrm{mmol} / \mathrm{l})$. TIEF was abnormal showing a type 2 pattern. Blood creatine kinase (CK) levels varied between 144 and $760 \mathrm{U} / \mathrm{l}$ and up to $4035 \mathrm{U} / \mathrm{l}$ in fever episodes. Histologic evaluation of the muscle biopsy showed no specific anomalies. ATP production from pyruvate oxidation was decreased, but the activity of the respiratory chain complexes I, II, IV and of the pyruvate dehydrogenase complex was normal. Prometaphase karyotype analysis, Multiplex Ligation-dependent Probe Amplification for subtelomeric microdeletions, mutation analysis of the LCAM1 locus and FISH analysis for Prader Willi syndrome were normal.

The child had a severe growth delay and failure to thrive (weight: $4060 \mathrm{~g}(<\mathrm{P} 3=7000 \mathrm{~g})$, length: $53 \mathrm{~cm}(<\mathrm{P} 3=64,5 \mathrm{~cm})$ and head circumference: $34 \mathrm{~cm}(<\mathrm{P} 3=42,5 \mathrm{~cm}))$, when she died at the age of 9 months.

Patient 2 was born at term as the third, male child of the same parents. At birth weight was $2890 \mathrm{~g}$ (25th centile), length $42 \mathrm{~cm} \quad(<3 \mathrm{rd}$ centile) and head circumference $33.5 \mathrm{~cm}$ (10th centile). A narrow and flat forehead with a low frontal hairline, short palpebral fissures, hypertelorism, a flat malar region, a wide, and short nose, a small mouth, retrognathia, low-set ears and a short neck with wrinkled skin (Figure 1) were noted. He had adducted thumbs, a simian crease, overlapping fingers with contractures of the PIP joints and ulnar deviation of the hands. He had a decreased muscle mass and muscle tonus with decreased deep tendon reflexes. The newborn needed further hospitalization secondary to severe pulmonary 

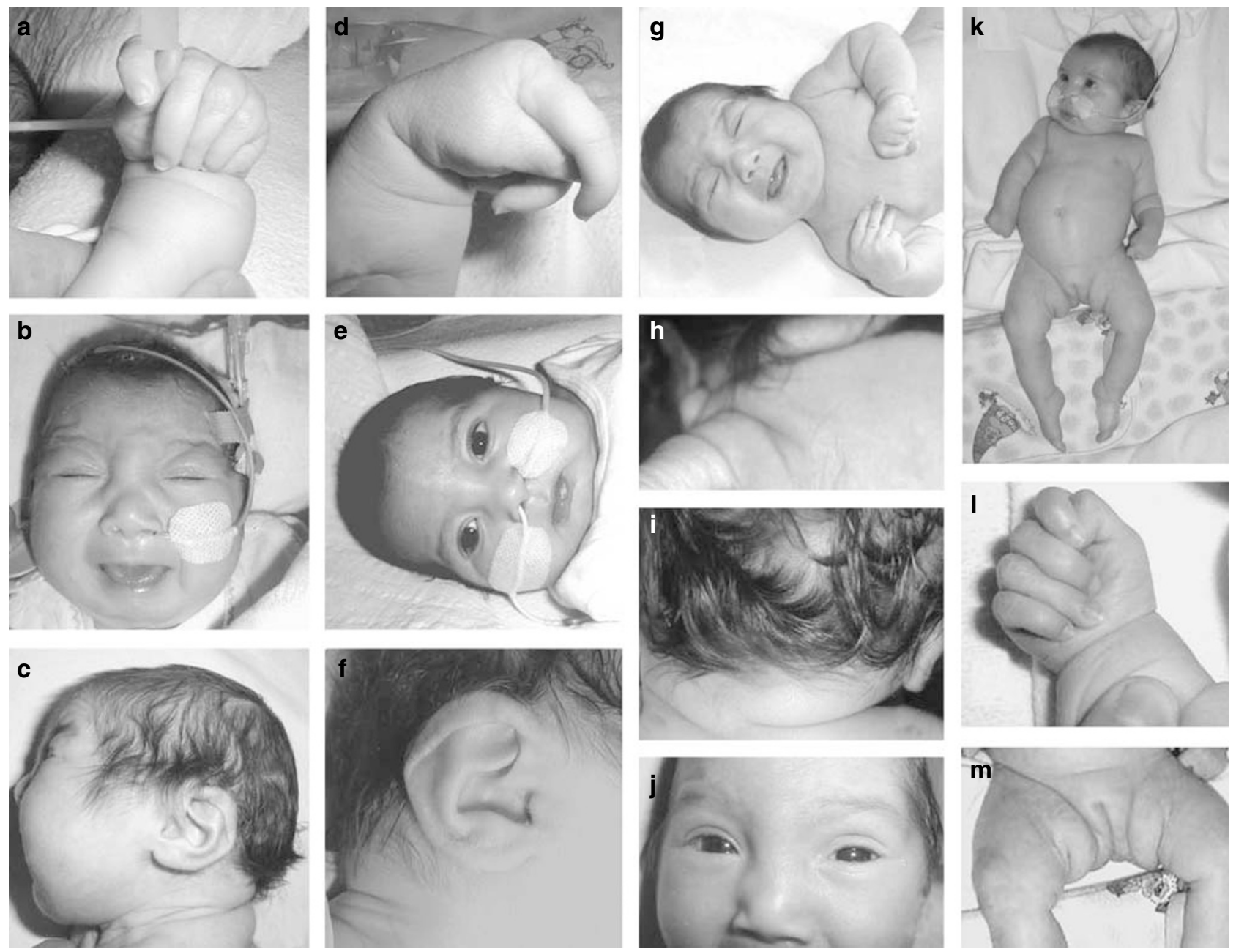

Figure 1 Clinical features of Patients $1(\mathbf{a}-\mathbf{d}, \mathbf{h}, \mathbf{j}, \mathbf{m})$ and $2(\mathbf{e}-\mathbf{g}, \mathbf{i}, \mathbf{k}, \mathbf{l})$. Note the profound microcephaly, flat forehead, low-set, dysplastic ears, short nose, retrognathia (b, $\mathbf{c}, \mathbf{e}, \mathbf{f}, \mathbf{g}, \mathbf{j})$. (h, i) Wrinkled skin around the neck and the posterior axillar region. (a, $\mathbf{d}$ and $\mathbf{g})$ adducted thumb and peripheral contractures of the DIP and PIP joint with overlapping fingers. (c) Note adducted thumb at the age of 7 months. (k and $\mathbf{m})$ Abnormal fat distribution in the patients around the thighs.

hypertension and urosepsis owing to obstructive uropathy. Blood CK levels (normal value $<200 \mathrm{U} / \mathrm{l}$ ) varied between 180 and $360 \mathrm{U} / \mathrm{l}$ and up to $4000 \mathrm{U} / \mathrm{l}$ in fever episodes. He received tube feeding for gastrointestinal pseudoobstruction. He also had a severe multisystem disorder, comparable with that of his older sister, including microcephaly, dysmorphic features, hypotonia with peripheral joint contractures. Brain ultrasound and MRI showed brain atrophy with cerebellar atrophy and corpus callosum hypoplasia. Kidney ultrasound showed hydronephrosis on the left. Ophthalmologic examination was normal. Echocardiography detected a peri-membranous VSD with ASD II (Table 1).

Laboratory investigations showed no signs of liver dysfunction or endocrine anomalies. The serum amino acids, acyl-carnitine profile, very long chain fatty acids, 7-dehydrocholesterol and urine organic acids were also normal. Blood CK activity was repeatedly elevated. The activities of several lysosomal enzymes (fucosidase, hexosaminidase and $\alpha$-mannosidase) were mildly elevated in plasma. TIEF showed a type 2 pattern. The patient died at the age of 7 months.

Patient 3 was born at term as the second female child of healthy, consanguineous parents (first cousins) of Maroccan origin. At birth weight was $2300 \mathrm{~g}(<3$ rd centile), length $41 \mathrm{~cm}(<3 \mathrm{rd}$ centile) and head circumference $31.9 \mathrm{~cm}(<3 \mathrm{rd}$ centile). Dysmorphic features were noted, including upslanting and short palpebral fissures, a narrow and flat forehead, a flat malar region, a wide, and short nose, a smooth phyltrum, a small mouth, retrognathia, thick gums, low-set, posteriorly-rotated ears and a short neck with wrinkled skin (Figure 2). She had adducted 
Table 1 Clinical features of our patients in comparison with those of the siblings described with $\operatorname{COG} 7,,^{2,7} \mathrm{COG}^{8}$ and COG $8^{17,18}$ deficiencies

\begin{tabular}{|c|c|c|c|c|c|c|c|}
\hline Clinical features & Patient 1 & Patient 2 & Patient 3 & $\begin{array}{l}\text { Wu et al and } \\
\text { Spaapen et al }\end{array}$ & $\begin{array}{l}\text { Wu et al and } \\
\text { Spaapen et al }\end{array}$ & $\begin{array}{l}\text { COG1 } \\
\text { Foulquier } \\
\text { et } a l^{8}\end{array}$ & $\begin{array}{l}\quad \text { COG8 } \\
\text { Briones et al } \\
\text { and Foulquier } \\
\text { et al }\end{array}$ \\
\hline Consanguinity & + & + & + & + & + & + & + \\
\hline Ethnicity & Morocco & Morocco & Morocco & Tunisia & Tunisia & Portugal & Spain \\
\hline Short length at birth & + & + & + & + & + & - & - \\
\hline Microcephaly at birth & + & - & + & + & + & - & - \\
\hline Progressive microcephaly & + & + & + & + & + & + & + \\
\hline Micrognathia & + & + & + & + & + & - & - \\
\hline Small mouth, retrognathia & + & + & + & + & + & - & + \\
\hline Short neck & + & + & + & + & + & - & + \\
\hline Wrinkled, loose, soft skin & + & + & + & + & + & - & - \\
\hline Adducted thumbs & + & + & + & + & + & - & - \\
\hline Overlapping, long fingers & + & + & + & + & + & - & + \\
\hline $\begin{array}{l}\text { Absence of humerus/tibia } \\
\text { epiphyses }\end{array}$ & - & - & - & + & ND & - & - \\
\hline Hypotonia & + & + & + & + & + & + & + \\
\hline Convulsions & + & - & + & + & ND & - & + \\
\hline Cerebral atrophy & + & + & + & + & + & + & + \\
\hline Hypoplasia of corpus callosum & + & + & + & - & - & - & - \\
\hline Periods with hyperthermia & + & + & + & + & + & - & - \\
\hline Perimembranous VSD, ASD II & + & + & - & - & + & - & - \\
\hline $\begin{array}{l}\text { Feeding problems/ } \\
\text { pseudoobstruction }\end{array}$ & + & + & - & + & ND & + & + \\
\hline Failure to thrive & + & + & + & + & ND & + & + \\
\hline CK elevations in blood & + & + & + & ND & ND & ND & + \\
\hline Liver enzyme elevations $^{a}$ & $+1-$ & $+1-$ & + & + & + & + & + \\
\hline Lethality in the first year & + & + & + & + & + & - & - \\
\hline
\end{tabular}

ND: not determined.

${ }^{a}$ Elevated ASAT/ALAT levels.

thumbs and overlapping, long toes and fingers. Axial tone was increased with tendency to opisthotonus. Brain MRI showed corpus callosum hypoplasia, wide cerebral ventricles and cortical-subcortical atrophy. The skeletal survey, liver ultrasound and ophthalmologic examination were normal. The echocardiogram was normal. On EEG, there were paroxysmal changes with multi-focal sharp spikes.

At the age of 3 weeks, she was admitted with extreme temperature elevation up to $41^{\circ} \mathrm{C}$ without proven infection. These episodes were repeated over the first year. Serology for perinatal infections was negative. By further screening laboratory investigations neither signs of kidney or liver dysfunction, nor endocrine or metabolic anomalies were found. During the course of the disease, the ASAT and ALAT levels became gradually elevated to 200-350 U/1 with increase of liver size to $3 \mathrm{~cm}$ under the costal margin. The serum amino acids, acyl-carnitine profile, very long chain fatty acids, 7-dehydrocholesterol were normal and urine organic acids showed increased ethylmalonic aciduria (four times elevated compared with controls). TIEF was not performed. Blood CK levels were always elevated and increased during temperature elevations, once up to $45.900 \mathrm{U} / 1$.
Histologic evaluation of the muscle biopsy showed no specific anomalies. Respiratory chain complexes I, II, IV and of the pyruvate dehydrogenase complex were normal. Prometaphase karyotype analysis, Multiplex Ligationdependent Probe Amplification for subtelomeric microdeletions were normal.

The child continued to have a failure to thrive, no developmental progress and episodes of extreme hyperthermia up to $42^{\circ} \mathrm{C}$ associated with increased of ASAT, ALAT, LDH and CK. She died at the age of 8 months.

\section{TIEF and ApoC-III}

Transferrin is an N-glycosylated protein. Multiple isoforms occur in plasma with the tetrasialo-isoform being the most abundant. TIEF is used as a screening method for the detection of N-glycosylation defects. ApoC-III is an Oglycosylated plasma protein with equal amounts of disialoand monosialo-isoforms in addition to a very small amount of asialo-ApoC-III. ApoC-III isofocusing is used as a screening method for the detection of mucin-type core 1 O-glycan biosynthesis defects. The methods were carried out as described previously. ${ }^{9-11}$ 

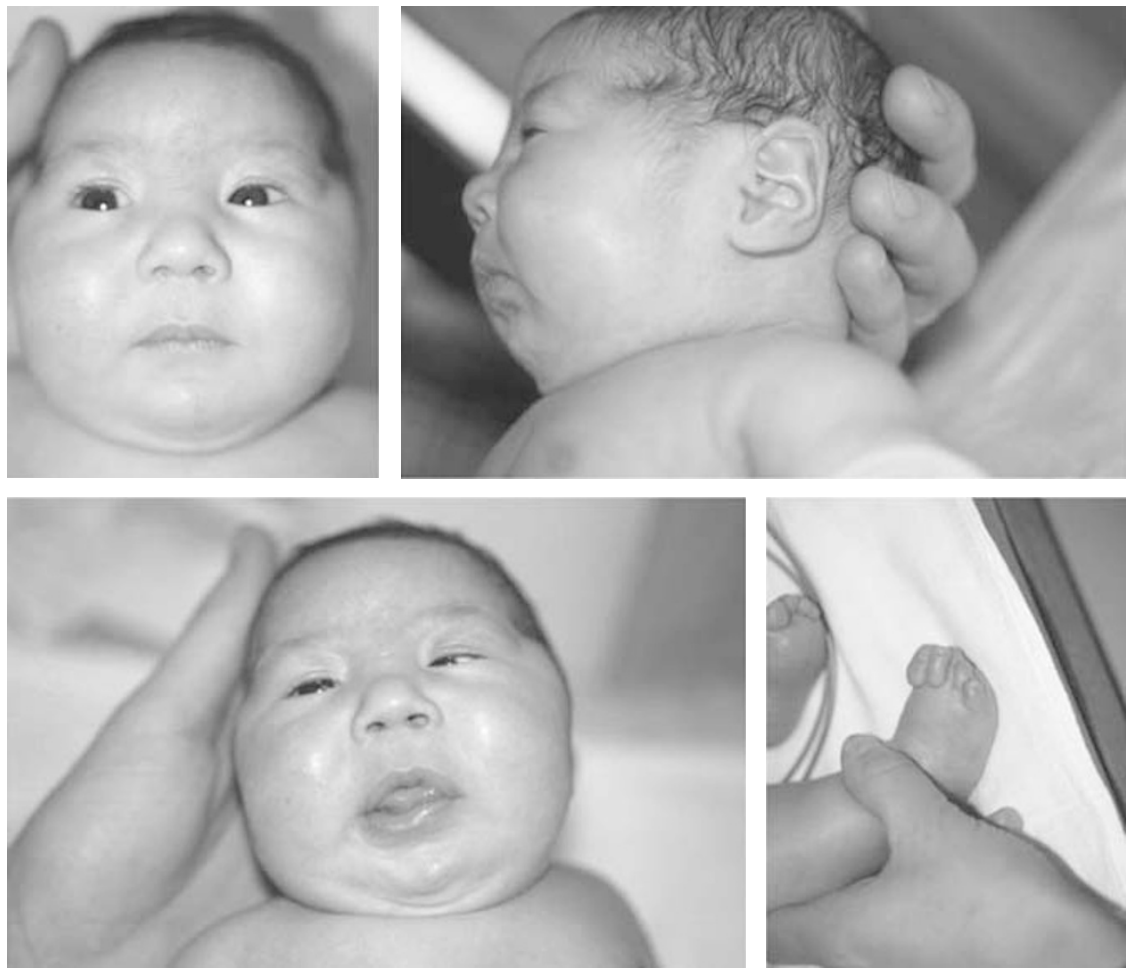

Figure 2 Clinical features of Patient 3 demonstrating microcephaly, flat forehead, dysplastic ears, short nose, retrognathia and irregular insertion of the toes.

\section{Western blot analysis of the COG subunits}

Control and patient's fibroblasts were rinsed twice with icecold PBS and lysed on ice in cell lysis buffer (Tris-buffered saline supplemented with 1\% Triton X-100 (Sigma, Bornem, Belgium) with protease inhibitor (Roche, Vilvoorde, Belgium) for $30 \mathrm{~min}$. Insoluble material was removed by centrifugation at $13000 \mathrm{~g}$ for $15 \mathrm{~min}$ at $4^{\circ} \mathrm{C}$. Proteins were quantified using the BCA kit (Pierce, Polylab, Merksem, Belgium). Equal quantities of protein were mixed with reducing $4 \times$ sample loading buffer (Invitrogen, Merelbeke, Belgium) containing 3\% mercapto-ethanol (Sigma), incubated at $90^{\circ} \mathrm{C}$ for $5 \mathrm{~min}$ and separated on $4-12 \%$ precast gels (Invitrogen). Proteins were transferred on nitrocellulose membranes (Amersham, Diegem, Belgium) and nonspecific binding sites were blocked by incubation in TBS containing $0.1 \%(\mathrm{v} / \mathrm{v})$ Tween-20 (Sigma) and 5\%(w/v) nonfat dried milk. Further processing of the Western blot was performed by application of the appropriate primary and horseradish peroxidase-conjugated secondary antibodies in the same blocking buffer. The blots were developed using chemiluminiscence (Renaissance, Perkin Elmer, Zaventem, Belgium) and the signals of the different subunits were quantified using Image J software (http://rsb.info.nih.gov/ij/).

\section{Molecular genetic/sequence analysis}

Mutation analysis on the index patients 1 and 3 was performed on cDNA for the genes COG2, COG3, COG4,
COG5, COG6, COG7 and on genomic DNA for COG1 and COG8. Total RNA was isolated from patient's fibroblasts and a normal control using the RNeasy Kit (QIAGEN, Venlo, The Netherlands). The cDNA was then prepared with oligo-dT priming (Amersham) and Superscript III RNase (Invitrogen). Primers were designed to amplify the entire cDNA, for example, COG7 primers forward (CCGGAGCCAACTCTTAAGTTC) and reverse (GAAGA GATTCGGCAGCAAGT). Other primer sequences are available on request.

A $0.4 \mu \mathrm{l}$ cDNA sample was used in a total volume of $50 \mu \mathrm{l}$ with DNA polymerase mix and buffer 1 of the expand long template PCR system (Roche). Amplification conditions were $2 \mathrm{~min}$ at $95^{\circ} \mathrm{C}, 10$ cycles of $10 \mathrm{~s}$ at $95^{\circ} \mathrm{C}, 30 \mathrm{~s}$ at $65^{\circ} \mathrm{C}$ $\left(-1^{\circ} \mathrm{C}\right.$ each cycle) and $2 \mathrm{~min}$ at $68^{\circ} \mathrm{C}$ followed by 25 cycles of $10 \mathrm{~s}$ at $95^{\circ} \mathrm{C}, 30 \mathrm{~s}$ at $55^{\circ} \mathrm{C}$ and $2 \mathrm{~min}$ at $68^{\circ} \mathrm{C}$.

Sequence analysis of the cDNA was performed in seven parts with overlapping ends using Big Dye Terminator v3.1 cycle sequencing kit on ABI 3100 Avant (Applied Biosystems, Nieuwerkerk a/d Ijsel, The Netherlands).

To confirm the presence of the COG7 mutation, exon 1 and the first part of intron 1 of the genomic DNA were amplified using the following primers: CCGGAGC CAACTCTTAAGTTC (F) and AGGGTATTTGCTGTCCATGC (R). Amplification conditions were $5 \mathrm{~min}$ at $95^{\circ} \mathrm{C}, 20$ cycles of $30 \mathrm{~s}$ at $95^{\circ} \mathrm{C}, 30 \mathrm{~s}$ at $65^{\circ} \mathrm{C}\left(-0,5^{\circ} \mathrm{C}\right.$ per cycle $)$ and $30 \mathrm{~s}$ at $72^{\circ} \mathrm{C}$ followed by 20 cycles of $30 \mathrm{~s}$ at $95^{\circ} \mathrm{C}, 30 \mathrm{~s}$ at $55^{\circ} \mathrm{C}$ and 
$30 \mathrm{~s}$ at $72^{\circ} \mathrm{C}$. These conditions were also used for amplification of the exons and flanking intronic regions of COG1 and COG8. The DNA was isolated from fibroblasts of patients 1 and 3, and from leucocytes for patient 2 .

\section{Haplotype analysis}

Polymorphic markers (D16S412, D16S403, D16S417, D16S481), spanning a region of $800 \mathrm{~kb}$ around COG7, were used to elucidate the haplotype of the family members and a member of the first described family with a COG7 defect. $^{2}$ The PCR product $(0.1 \mu \mathrm{l})$ was mixed with $0.1 \mu \mathrm{l}$ Gene Scan 500 ROX size standard (Applied Biosystem) and $10 \mu \mathrm{l} \mathrm{Hi-Di}$ formamide (Applied Biosystems) and was put for $3 \mathrm{~min}$ on $95^{\circ} \mathrm{C}$. Analysis was performed with Gene Scan 3.7 (Applied Biosystems).

\section{Results}

\section{Transferrin isoelectric focusing}

Plasma samples from the patients (Patients 1 and 2, respectively) showed repeatedly an abnormal transferrin isofocusing pattern with a decrease of the tetrasialotransferrin isoforms (23 and 30\%, controls: 52-70\%), but increase of hypoglycosylated transferrin isoforms (Figure 3) in both siblings; asialo: 12 and 3\%, controls: $<3 \%$; monosialo: 12 and $8 \%$, controls <4\%; disialo: both $17 \%$, controls: $<3-11 \%$, trisialo: 24 and $26 \%$, controls: $4-17 \%)$. A protein polymorfism as the cause of an abnormal transferrin isofocusing profile was excluded by sialidase digestion. The presence of sialidase in the blood samples of both patients (either due to certain infections or improper blood sampling/handling) was excluded by enzyme activity measurement in serum, which showed no activity. The patients were classified as having an unidentified CDG type
II, suggesting that the defect is situated in the processing of N-glycans localized in the Golgi. No analysis was performed in Patient 3.

\section{ApoC-III isoelectric focusing}

Plasma samples from the two patients analysed showed repeatedly an abnormal ApoC-III isofocusing profile (Figure 2). Patients 1 and 2, respectively, had the characteristic pattern of increased amounts of ApoC- $\mathrm{III}_{0}$ (17 and 12\%, controls: $0-8 \%$ ), normal amounts of ApoC-III 1 (57 and 66\%, controls: $34-58 \%$ ) and decreased amounts of ApoC$\mathrm{III}_{2}$ (26 and 22\%, controls: 40-62\%). The ApoC-III isoform distribution was comparable with one of the patients described with a CDG-IIe defect (COG7 in Figure 2. ${ }^{2,7,10}$ )

\section{Western blot analysis of the COG subunits}

Compared with controls a significant decrease in the steady state levels of the lobe B subunits was found in Patients 1 and 2. Semiquantitative analysis of the reduced subunits showed 94\% decrease for COG5, 73\% decrease for COG6, 95\% decrease for COG7 and 71\% decrease for COG8 (Figure $3 \mathrm{~b}$ ). These results are in accordance with results from previous experiments in COG7-deficient mammalian cells.

\section{Genetic analysis}

Genetic analysis of the COG7 was performed in Patients 1 and 2 based on the biochemical findings, and in Patient 3 based on the characteristic phenotype. Mutation analysis on cDNA performed on patients 1 and 3 revealed at least two different transcripts, one with a 19 base pair deletion and one with a 83 base pair insertion. Sequence analysis of exon 1 and the flanking intronic sequence on genomic DNA showed a homozygous intronic splice site mutation c. $169+4 \mathrm{~A}>\mathrm{C}$. This mutation disrupts the splice donor site

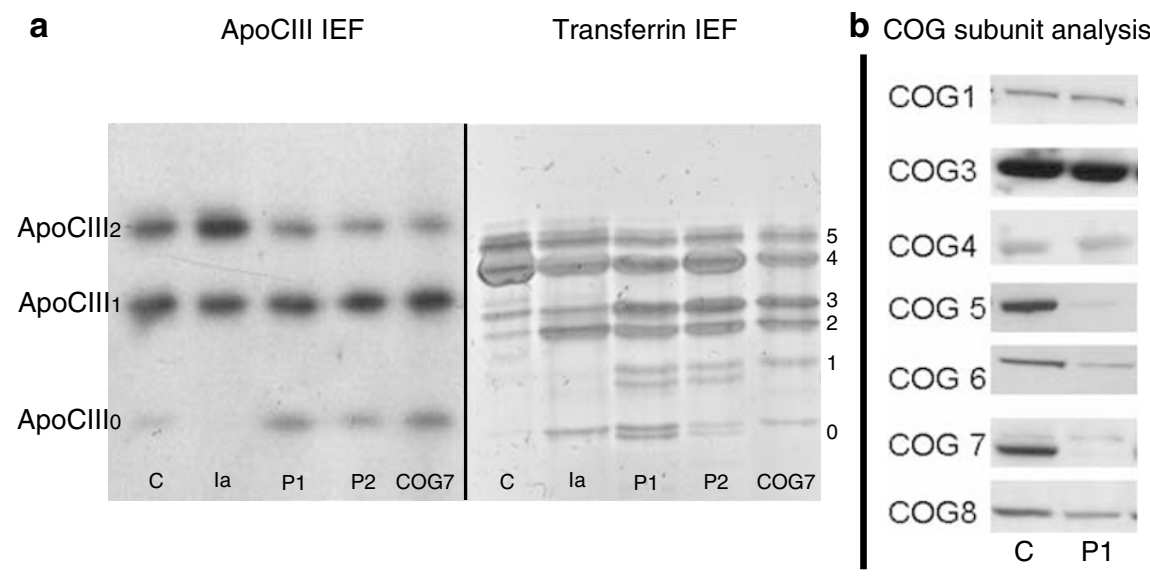

Figure 3 (a) Isoelectric focusing of ApoC-III and transferrin in Patients 1 and 2 compared with healthy controls, to the pattern in CDG type la, and the results of the child described by Freeze et al (COG7; Wu et $a l^{2}$ and Spaapen et $a l^{7}$ ) carrying the same genetic and biochemical defect, demonstrating hypoglycosylation of both mucin core 1 O-glycans and N-glycans. (b) COG subunit analysis in Patient 1 shows COG7 deficiency with secondary deficiency of the associated lobe B subunits. 
and activates at least two different cryptic splice sites, leading to the different transcripts. The same mutation was found in patient 2 (sibling of patient 1), and in Patient 3. The parents and a healthy sibling of patients 1 and 2 were heterozygous for the c. $169+4 \mathrm{~A}>\mathrm{C}$ mutation. Mutation analysis of the other subunits of the COG complex in patients 1 and 3 , did not detect any other pathogenic mutations.

Haplotyping with four polymorphic markers in the neighbourhood of COG7 showed that all three affected children were homozygous for the same haplotype of $680 \mathrm{~kb}$ around the COG7 gene (polymorphic markers D16S481 and D16S417). The previously described COG7 patient 5 (Table 1) from the study of Wu et $a l^{2}$ had the same mutation and the same haplotype as the present patients. This patient is homozygous for the same haplotype of $800 \mathrm{~kb}$ with patients 1 and 2 around the COG7 gene (all four polymorphic markers).

\section{Discussion}

Patients with a serum transferrin IEF type 2 pattern, can be divided in subgroups, namely with normal and abnormal ApoC-III IEF profiles. ${ }^{10}$ In our initial patients, a type 2 transferrin pattern was found together with an abnormal ApoC-III 0 profile. All COG-deficient patients, so far, show the same combination of IEF profiles. The finding of the characteristic biochemical 'pattern' by the initial metabolic screening could be very important in the diagnostics of patients with a suspected defect of the COG complex.

Here, we report on three additional patients with a defect in the COG7 subunit of the COG complex. Intriguingly, the patients carry the same homozygous mutation c. $169+4$ A $>\mathrm{C}$ as those described previously. ${ }^{2}$ The c. $169+4 \mathrm{~A}>\mathrm{C}$ point mutation impairs splicing at the canonical site and allows the use of a cryptic, conserved alternative splicing site near the first exon/intron boundary. One might consider the possibility of a mutation hot spot as the underlying aetiology for the common mutation. No known trinucleotide or tetranucleotide motifs are positioned at the mutation site, which have been proven to increase the frequency of this particular mutation. ${ }^{12}$

Furthermore, according to our extended family history, the members of the three families carrying the COG7 mutations are not related. Interestingly, all families relocated from Africa; the patients described by Wu et $a l^{2}$ originated from Tunisia, whereas our patients are of Moroccan ancestry. The mutation presents within the same haplotype in all the three, so far described families, confirmed by haplotype analysis with markers spanning $680 \mathrm{~kb}$ around the COG7 gene. The common ethnic and genetic ancestry of large subgroups of Tunisian and Moroccan population could give an explanation for the overlapping haplotypes and the patients may share the same ancestral mutation. ${ }^{13}$ This specific mutation could be a common North African mutation, however, this needs further investigations.

The phenotypic features of the two patients are progressive microcephaly, characteristic facial features, intrauterine growth retardation, feeding difficulties owing to intestinal pseudoobstruction with failure to thrive, pronounced congenital hypotonia with adducted thumbs, cardiac anomalies, wrinkled skin and episodes of severe hyperthermia. The combination of profound microcephaly, adducted thumbs, heart defects and idiopathic hyperthermia is rather unique, and seldom reported in children. Neonatal hypotonia, distal finger contractures/ adducted thumbs and severe microcephaly have been described in patients with the adducted thumb syndrome (MIM 201550), which appears to be genetically heterogeneous. Our patients have many overlapping features with this syndrome, including the neonatal hypotonia, finger anomalies, microcephaly, ear anomalies, telecanthus, flat forehead, seizures and early death. Most patients however have no cardiac defects, but craniosynostosis or congenital brain malformations with contractures of the large joints, which were not present in the two siblings. ${ }^{14,15}$ A similar clinical phenotype is observed in children with a 1p36.3 chromosomal microdeletion, including hypotonia, neonatal short stature, growth delay, microcephaly, adducted thumbs, ear abnormalities, short palpebral fissures and microcephaly. ${ }^{16}$ High-resolution chromosome analysis and MLPA studies for possible subterminal microdeletions, however, were normal in our patients.

Comparing the clinical features of the siblings with those previously described with a COG deficiency, we have found some overlapping features with children carrying mutations in the COG1 gene, including the multisystem involvement, hypotonia, growth delay, short stature, microcephaly, dysmorphic features and the hepatic involvement ${ }^{8}$ (Table 1). A somewhat similar clinical spectrum of symptoms has been observed in a patient with COG8 deficiency, including hypotonia, growth and developmental delay, short stature, strabismus and ataxia, comparable with the phenotype, observed in children with mitochondrial disorders (Table 1). ${ }^{17,18}$ However, the children described with COG1 and with COG8 deficiency had a significantly milder clinical presentation. The proband with COG1 mutation had associated ventricular hypertrophy/ cardiac dysfunction and a rhizomelic short stature as well not present in our patients.

Regarding the patients described with the same COG7 mutation, we confirmed a consistent phenotype. The most pronounced difference compared with our patients was the presence of skeletal anomalies in the previously reported patients and a less pronounced liver involvement in our patients. Our patients on the other hand, had episodes of hypoglycemia, and severe recurrent episodes with 
hyperthermia. We propose that the few differences observed are comparable with the intra-familial variability seen in various metabolic disorders.

Our third patient was diagnosed without any previous metabolic screening, simply based on the characteristic clinical presentation especially the episodes of extreme hyperthermia. The presence of the suggestive phenotype therefore might warrant direct DNA analysis and could be sufficient to diagnose this new genetic syndrome.

\section{Acknowledgements}

The work was supported by the European Commission (FPG, contract No 512131 (EUROGLYCANET)). Renate Zeevaert is a PhD fellow for the FWO, Vlaanderen. Further special thanks for the unpublished information provided by Leo Spaapen, and to Monty Krieger, Daniel Ungar and Vladimir Lupashin for providing antibodies.

\section{References}

1 Jaeken J, Vanderschueren-Lodeweycks M, Casaer P et al: Familial psychomotor retardation with markedly fluctuating serum proteins, FSH and GH levels, partial TBG-deficiency, increased serum arylsulphatase A and increased CSF proteins: a new syndrome? Pediatr Res 1980; 14: 179.

$2 \mathrm{Wu}$ X, Steet R-A, Bohorov O et al: Mutation of the COG complex subunit gene COG7 causes a lethal congenital disorder. Nat Med 2004; 10: 518-523.

3 Ungar D, Oka T, Brittle E-E et al: Characterization of a mammalian Golgi-localized protein complex, COG, that is required for normal Golgi morphology and function. J Cell Biol 2002; 157: $405-415$.

4 Loh E, Hong W: The binary interacting network of the conserved oligomeric Golgi tethering complex. J Biol Chem 2004; 279: $24640-24648$.

5 Shestakova A, Zolov S, Lupashin V: COG complex-mediated recycling of Golgi glycosyltransferases is essential for normal protein glycosylation. Traffic 2006; 7: 191-204.

6 Oka T, Vasile E, Penman M et al: Genetic analysis of the subunit organization and function of the conserved oligomeric golgi (COG) complex: studies of COG5- and COG7-deficient mammalian cells. J Biol Chem 2005; 280: 32736-32745.
7 Spaapen L-J, Bakker J-A, van der Meer S-B et al: Clinical and biochemical presentation of siblings with COG-7 deficiency, a lethal multiple $\mathrm{O}$ - and $\mathrm{N}$-glycosylation disorder. J Inherit Metab Dis 2005; 28: 707-714.

8 Foulquier F, Vasile E, Schollen E et al: Conserved oligomeric Golgi complex subunit 1 deficiency reveals a previously uncharacterized congenital disorder of glycosylation type II. Proc Natl Acad Sci USA 2006; 103: 3764-3769.

9 Wopereis S, Grunewald S, Morava E et al: Apolipoprotein C-III isofocusing in the diagnosis of genetic defects in O-glycan biosynthesis. Clin Chem 2003; 49: 1839-1845.

10 Wopereis S, Morava E, Grunewald S et al: Patients with unsolved congenital disorders of glycosylation type II can be subdivided in six distinct biochemical groups. Glycobiology 2005; 15: $1312-1319$

11 Morava E, Wopereis S, Coucke $\mathrm{P}$ et al: Defective protein glycosylation in patients with cutis laxa syndrome. Eur J Hum Genet 2005; 13: 414-421.

12 Antonarakis SE, Krawczak M, Cooper DN: The nature and mechanisms of human gene mutation; in: Scriver CR, Beaudet AL, Sly WS, Valle D (eds):: The metabolic \& molecular bases of inherited disease. New York: McGraw-Hill, 2001, 8th edn. pp $343-377$.

13 Cherni L, Loueslati BY, Pereira L, Ennafaa H, Amorim A, El Gaaied $\mathrm{AB}$ : Female gene pools of Berber and Arab neighboring communities in central Tunisia: microstructure of mtDNA variation in North Africa Hum Biol, 2005; 77: 61-70.

14 Christian J-C, Andrews P-A, Conneally P-M, Muller J: The adducted thumbs syndrome. An autosomal recessive disease with arthrogryposis, dysmyelination, craniostenosis, and cleft palate. Clin Genet 1971; 2: 95-103.

15 Moldavsky M, Lerman-Sagie T, Kutai M, Legum C, Harel S: Heterogeneity in adducted thumbs sequence. Am J Med Genet 1977; 70: 114-117.

16 Finelli P, Giardino D, Russo S et al: Refined FISH characterization of a de novo $1 \mathrm{p} 22-\mathrm{p} 36.2$ paracentric inversion and associated 1p21-22 deletion in a patient with signs of 1 p36 microdeletion syndrome. Am J Med Genet 2001; 99: 308-313.

17 Briones P, Vilaseca M-A, Garcia-Silva M-T et al: Congenital disorders of glycosylation (CDG) may be underdiagnosed when mimicking mitochondrial disease. Eur J Paediatr Neurol 2001; 5: $127-131$.

18 Foulquier F, Ungar D, Reynders E et al: A new inborn error of glycosylation due to $\operatorname{Cog} 8$ deficiency reveals a critical role for the Cog1-Cog8 interaction in COG complex formation. Hum Mol Genet 2007 January 12, (E-pub ahead of print). 